\title{
Association of Diaphragmatic Surgery as Part of Cytoreductive Effort in Advanced Stage Ovarian Cancer
}

\author{
NICOLAE BACALBASA ${ }^{1}$, IRINA BALESCU ${ }^{2}$, CRISTIAN BALALAU ${ }^{1,3}$, \\ OLIVIA IONESCU ${ }^{4}$ and CLAUDIA STOICA ${ }^{5}$ \\ ${ }^{1}$ Department of Obstetrics and Gynecology "Carol Davila" \\ University of Medicine and Pharmacy, Bucharest, Romania; \\ ${ }^{2}$ Department of General Surgery "Ponderas" Academic Hospital, Bucharest, Romania; \\ ${ }^{3}$ Department of General Surgery, "Sf. Pantelimon" Clinical Hospital, Bucharest, Romania; \\ ${ }^{4}$ Department of Obstetrics and Gynecology "Fichtelgebirge", Clinic, Marktredwitz, Germany; \\ ${ }^{5}$ Department of General Surgery "Ilfov" Clinical County Hospital, Bucharest, Romania
}

\begin{abstract}
Background/Aim: Diaphragmatic surgery in advanced-stage ovarian cancer has been considered since long time to increase the rates of postoperative complications. However, improvement of surgical techniques and perioperative management of these patients has lead in the last decade to a safe association of such procedures as part of debulking process. The aim of the current paper was to report our experience regarding the role of diaphragmatic resections as part of debulking surgery for advanced stage ovarian cancer. Materials and Methods: Between 2014 and 2016 diaphragmatic surgery was performed in 22 cases with advanced stage ovarian cancer. Results: Diaphragmatic surgery consisted of diaphragmatic peritoneal resection in 10 cases, full thickness diaphragmatic resections in four cases and coagulation of peritoneal nodules in eight cases. In all but two cases debulking surgery to no residual disease was achieved. Other upper abdominal resections consisted of splenectomy - in four cases, liver resections - in three cases, glissonian capsule resections - in eight cases, distal pancreatectomy - in one case and partial gastrectomies in two cases. The postoperative outcomes were similar irrespective of type of diaphragmatic surgical procedure. Conclusion: Diaphragmatic surgery is a crucial procedure which can be safely associated as part of debulking surgery for advanced stage ovarian cancer.
\end{abstract}

This article is freely accessible online.

Correspondence to: Irina Balescu, Department of General Surgery "Ponderas" Academic Hospital, Bucharest, Romania. Tel: +40 724077709, e-mail: irina.balescu@ ponderas-ah.ro

Key Words: Advanced-stage ovarian cancer, diaphragmatic resection, diaphragmatic peritonectomy.
Diaphragmatic involvement in advanced stage ovarian cancer has been widely reported, the main mechanism of spread consisting of peritoneal dissemination. However, most cases presenting diaphragmatic involvement usually report the presence of other upper abdominal lesions, complete cytoreductive surgery necessitating multiple upper visceral resections $(1,2)$. Although it was initially considered that the presence of disseminated upper abdominal lesions is an indirect sign of a more aggressive biology of the tumor, more recent studies have infirmed that hypothesis and demonstrated that debulking surgery to no residual disease can provide a significant benefit in terms of survival in such cases too. Moreover, improvement of perioperative and postoperative management of these patients led to a safe association of extended upper abdominal resections, including diaphragmatic surgery without increasing the rate of postoperative morbidity (1-4).

\section{Materials and Methods}

Data of patients submitted to surgery for advanced stage ovarian cancer between 2014 and 2016 were retrospectively reviewed. Among the 135 patients submitted to surgery for advanced stage ovarian cancer, 22 patients necessitated diaphragmatic procedures. None of these patients received neoadjuvant chemotherapy. Preoperatively all patients were routinely submitted to computer tomography, chest X ray and determination of CA 125 levels, while the extent of the disease was classified according to the International Federation of Obstetrics and Gynecology (FIGO) system -2010 . All procedures were performed by the same team consisting of an oncogynecologist and an oncological surgeon and intended obtaining a complete cytoreduction. The completeness of cytoreductive procedures was defined as complete resection - if no residual disease was left in place, optimal resection - if lesions smaller than $0.5 \mathrm{~cm}$ were still present at the of surgery while suboptimal surgery was defined by the presence of lesions larger than $0.5 \mathrm{~cm}$. Diaphragmatic surgical procedures consisted of 
peritoneal stripping defined as the excision of the peritoneal layer from the subjacent muscle, full thickness resection defined as the resection of the peritoneum in association with the subjacent muscle and pleura or coagulation of the diaphragmatic lesions. Postoperative chest drainage tubes were left in place whenever full thickness resections larger than $5 \mathrm{~cm}$ were needed. Postoperatively all patients were submitted to routine chest $\mathrm{X}$ ray within the first three postoperative days.

\section{Results}

Between 2014 and 2016, 22 of the 135 patients submitted to surgery for advanced stage ovarian cancer necessitated performing diaphragmatic surgical procedures in order to maximize the debulking effort. The mean age at the time of introduction in the current study was 48 years (range $=33-67$ years). The clinico-pathological characteristics of the patients are summarized in Table I. Preoperative involvement of the diaphragm was visible in 12 of the 22 cases at the computed tomography.

All patients were submitted to total hysterectomy, bilateral adnexectomy, omentectomy, pelvic and para-aortic lymph node dissection, pelvic and parietal peritonectomy.

When it comes to the intraoperative outcomes of the diaphragmatic procedures, these consisted of diaphragmatic peritoneal resection in 10 cases, full thickness diaphragmatic resections in four cases and coagulation of peritoneal nodules in eight cases. Intraoperatively nine cases were diagnosed with bilateral diaphragmatic lesions; eight cases presented only right hemidiaphragmatic involvement while the other five cases presented only left hemidiaphragmatic lesions. Full thickness diaphragmatic resections resulted in defects larger than $5 \mathrm{~cm}$ in one case, while in all the other three cases smaller defects were encountered. However, in all cases in which a larger than $5 \mathrm{~cm}$ diaphragmatic resection was necessary a thoracic drainage tube was left in place. In all cases the resulting defect was sutured in an anatomical manner, no addition of mesh or prosthesis being needed. In 20 cases debulking surgery to no residual disease was achieved; in one case optimal cytoreduction was feasible while in the last case the cytoreduction was classified as suboptimal due to the presence of bulky tumoral lesions at the level of the suprahepatic veins. Other upper abdominal resections consisted of glissonian capsule resections - in eight cases, splenectomy in four cases, liver resections - in three cases, partial gastrectomies in two cases and distal pancreatectomy - in one case. In 14 of the 22 cases other digestive resections were needed and consisted of small bowel resections (in six cases), right colectomies (in four cases), rectosigmoidian resections (in three cases) and transverse colectomy respectively (in one case). Intraoperative aspects of the diaphragmatic surgical procedures are represented in Figures 1-6.

The mean blood loss was $550 \mathrm{ml}$ (range $=300-1100 \mathrm{ml}$ ) while the mean operative time was $330 \mathrm{~min}$ (range=220-450 $\mathrm{min}$ ).

Postoperatively, the most common encountered complication
Table I. Clinico-pathological characteristics of the patients submitted to diaphragmatic surgery as part of primary cytoreductive surgery.

\begin{tabular}{lc}
\hline All cases & 22 \\
Age (years), mean (range) & $48(33-67)$ \\
FIGO stage & \\
IIIC & 12 cases \\
IV & 10 cases \\
CA125 (UI/ml), median (range) & $834(145-2375)$ \\
Histopathological subtype & \\
Epithelial & 20 \\
Nonepithelial & 2 \\
Differentiation grade & 4 \\
G1 & 9 \\
G2 & 9 \\
G3 & 9 \\
\hline
\end{tabular}

consisted of pleural effusion and was reported in eight patients. Among these cases, a conservative management was performed in two cases, while the other six cases necessitated chest drainage through thoracocentesis (in two cases) or through thoracic tube placement (in the remnant four cases). When we analyzed the relationship between the type of surgery and development of postoperative pleural effusion, we observed that no connection between the surgical procedure and the development of pleural effusion can be established; among the eight patients diagnosed with this complication, the initial surgical procedure consisted of peritoneal stripping in four cases, full thickness resection in one case and peritoneal nodules coagulation in three cases. Other reported complications consisted of pneumothorax (in one case who had been submitted to coagulation of the peritoneal lesions) and pulmonary embolism (in one case who had necessitated an extended diaphragmatic resection). Non-related to diaphragmatic surgery complications consisted of one case of early postoperative hemoperitoneum and two cases of digestive leaks. Reoperation was necessary in the case that developed a postoperative hemoperitoneum and in one case that developed a digestive leak originating from a low colorectal anastomosis; in the latter case the anastomosis proved to be entirely compromised so a Hartmann procedure was performed. The other patient who developed a digestive fistula had been initially submitted to a right colectomy and the leak was successfully treated in a conservative manner. The median hospital stay was 13 days (range $=8-33$ days) while the median interval until the initiation of chemotherapy was 45 days (range $=30-55$ days). No early postoperative death was recorded.

\section{Discussion}

Diaphragmatic involvement in stage III-IV ovarian cancer is a common finding, being reported with an incidence of 40 up to $90 \%(1,2)$. Although it is a very common 


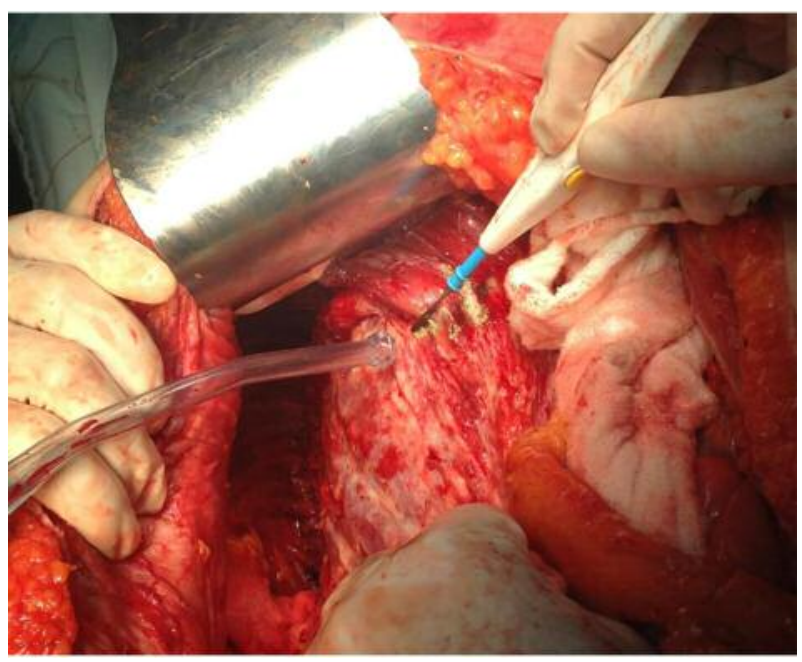

Figure 1. Peritoneal stripping of the right diaphragm.

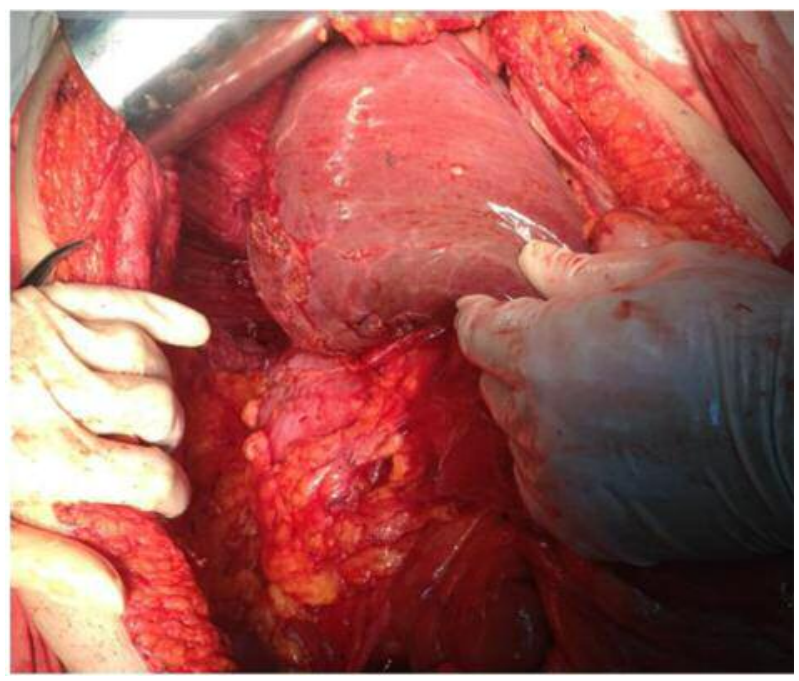

Figure 2. The final aspect of right diaphragmatic peritoneum resection. Complete mobilization of the liver was necessary.

intraoperative finding, diaphragmatic involvement is rarely diagnosed preoperatively, no specific radiological sign being described so far (5). In a study conducted in 2010 on 87 patients diagnosed intraoperatively with diaphragmatic involvement and advanced stage or recurrent ovarian cancer, the preoperative imaging studies could diagnose the extent of the disease at this level only in $42.5 \%$ of cases (6). The most commonly incriminated mechanism leading to the apparition of diaphragmatic lesions is throughout peritoneal dissemination or by direct contamination and have been

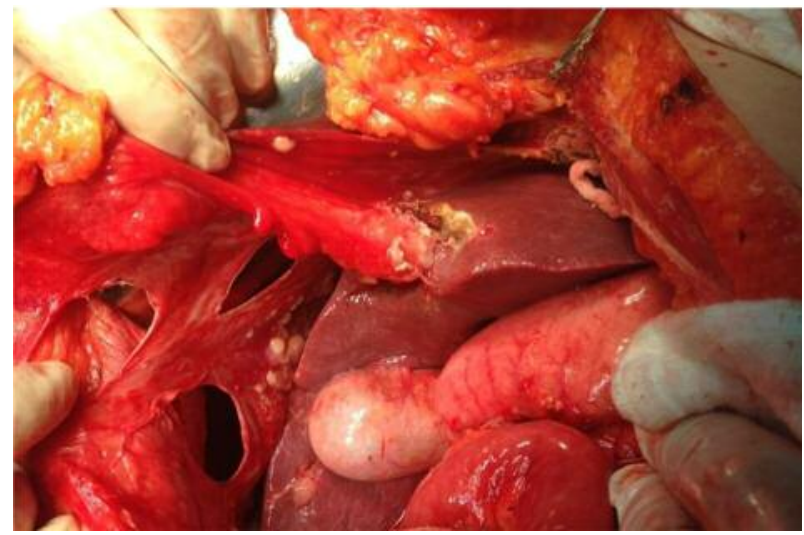

Figure 3. Diaphragmatic peritonectomy in association with Glissonian nodules resection.

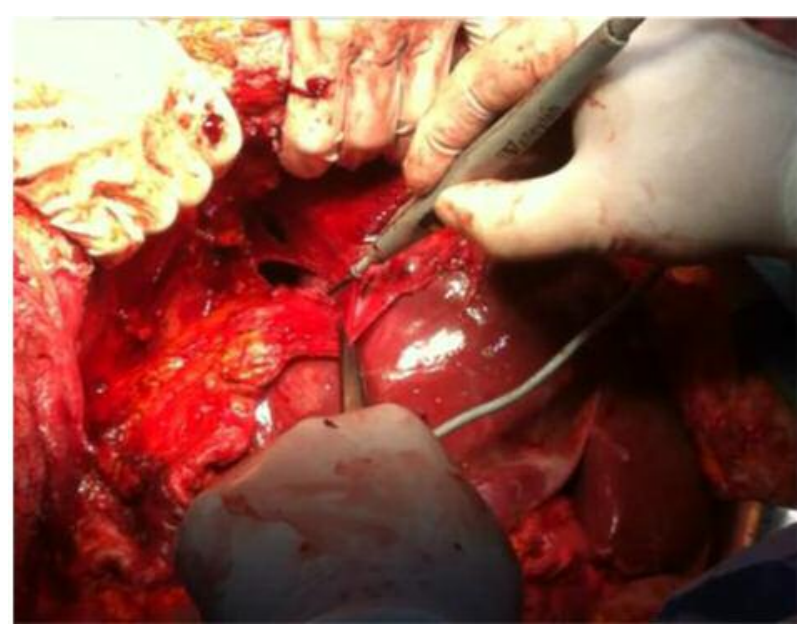

Figure 4. Full thickness diaphragmatic resection.

reported more frequently on the right hemidiaphragm, in association with other bulky upper abdominal lesions (6).

Traditionally it has been considered that approaching lesions located at the level of the diaphragmatic muscle is associated with significantly higher risks of perioperative morbidity; due to this reason, conservative therapeutic options have been proposed $(3,4)$. However, in the last decades studies have demonstrated that full thickness diaphragmatic resections can be safely associated as part of the debulking process without significant modifications of the perioperative outcomes $(5,7-9)$. Moreover, it has been proven that diaphragmatic resections can be safely associated as part of the extended upper abdominal resections in order to maximize the debulking effort $(5,7,10-16)$. One of the 


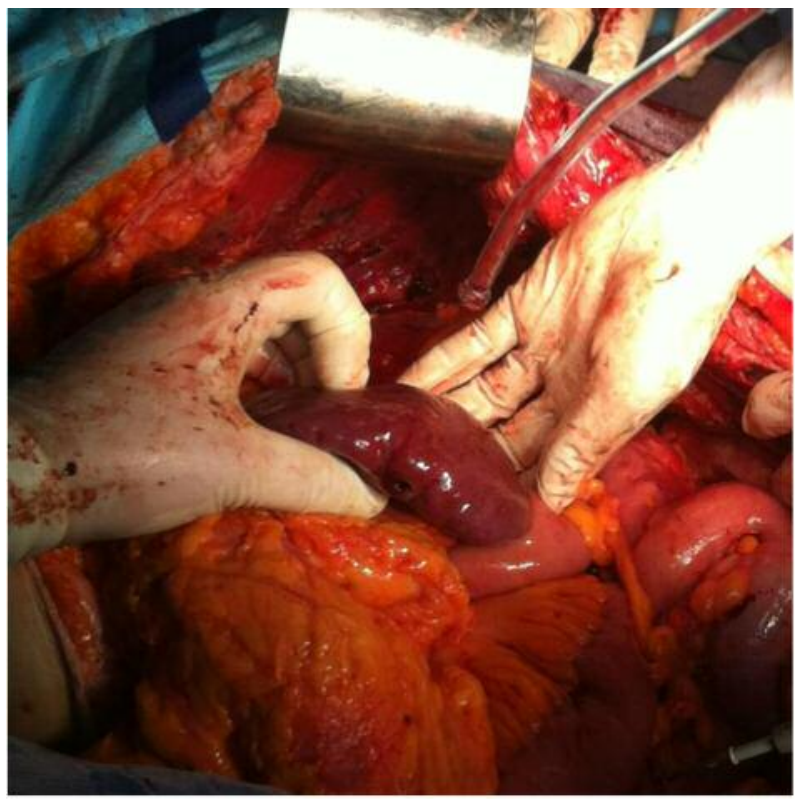

Figure 5. Left diaphragmatic peritonectomy. Complete mobilization of the spleen.

studies which come to sustain this point of view was conducted by Eisenkop et al. and published in 2001; the authors demonstrated that the long term postoperative outcomes of patients with advanced ovarian cancer was rather influenced by the completion of the cytoreductive procedures than by the initial extent of the disease (14).

When it comes to the most commonly performed diaphragmatic surgical procedures, they are represented by peritoneal stripping, peritoneal diaphragmatic resections, argon beam coagulation or radiofrequency ablation $(6,17,18)$. The choice of a certain therapeutic strategy is influenced by the extent of the disease and by the general status of the patient but it also seems to be influenced by the surgeon's experience and ability to determine the in-depth extension of the tumoral lesions (6). In cases presenting small diaphragmatic lesions, with no muscular involvement, argon beam coagulation might be tempted (19). When it comes to the risk of development of postoperative complications in patients submitted to diaphragmatic peritonectomy versus those submitted to full thickness resections, studies have shown that the type of procedure does not significantly impact on the perioperative outcomes. However, the histopathological studies confirmed that patients presenting larger macroscopic lesions submitted to full thickness resections usually present invasion in all the resected layers (including pleura) in up to $20 \%$ of cases (5).

Another parameter which might influence the type of procedure is the administration of neoadjuvant chemotherapy; sometimes in such cases it has been demonstrated that the

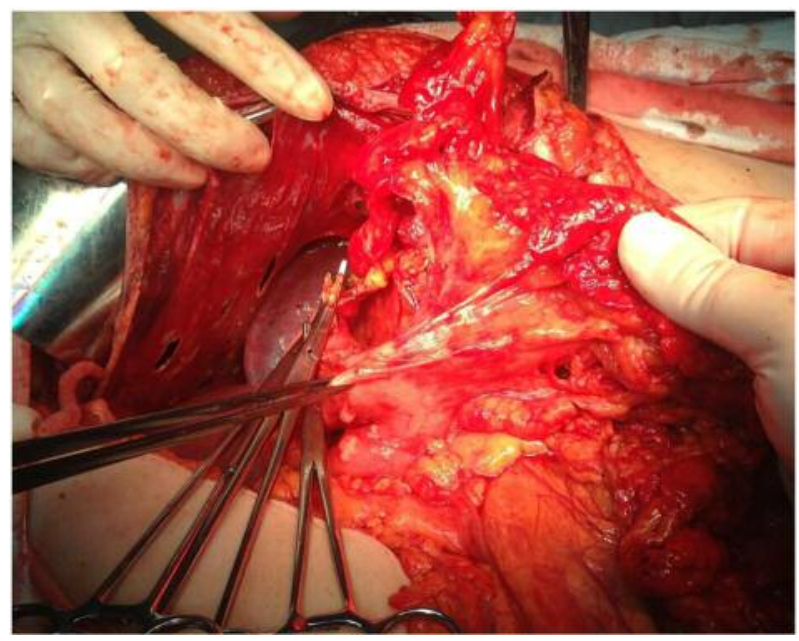

Figure 6. Left parietal and diaphragmatic peritoneal resection.

peritoneal stripping is harder to be performed due to the modifications of the tissular structures, higher bleeding rates being reported (6). However, the data regarding the negative effect of neoadjuvant chemotherapy on the perioperative outcomes in patients submitted to diaphragmatic surgical procedures are inconstant. One of the most representative studies regarding this issue was conducted in "Gustave Roussy" Institute, Paris, France between 2005 and 2008 and included 22 patients submitted per primam to surgery and 41 patients submitted to interval debulking surgery. The authors demonstrated that there was no significant difference in terms of the completion of cytoreduction or in type of performed diaphragmatic surgical procedure between the two groups. Moreover, administration of neoadjuvant chemotherapy did not increase the necessity of chest tube placement or of the perioperative morbidity rates. Although administration of neoadjuvant chemotherapy had been expected to decrease the extent of the disease and the necessity of performing bilateral diaphragmatic surgical procedures, this fact was not revealed by the intraoperative aspects (19).

However, other French study groups reported that the overall complication rates were significantly higher in patients submitted to per primam surgery when compared to the interval debulking surgery group, these data being explained through a higher percentage of complete cytoreductive surgical procedures among the first category (20).

Moreover, other authors demonstrated that diaphragmatic surgery can be safely performed even in cases diagnosed with recurrent ovarian cancer. One of these studies was conducted by Fanfani et al. and was published in 2010; the authors included 120 patients diagnosed with advanced stage ovarian cancer and submitted to primary cytoreduction, 74 patients 
submitted to interval debulking surgery and 40 patients submitted to surgery for ovarian cancer relapse. Among these cases, 87 patients were submitted to diaphragmatic surgery as part of primary cytoreduction (50 cases), as part of interval debulking surgery (in 16 cases) and as part of secondary cytoreductive surgery (in the remaining 21 cases). However, most cases were also submitted to other upper abdominal resections, the most commonly performed procedures including splenectomies (in $21.8 \%$ of cases), liver resections (in $9.2 \%$ of cases), distal pancreatectomies (in $3.4 \%$ of cases), cholecystectomies (in $6.9 \%$ of cases) and gastric resections (in $1.1 \%$ of cases). Moreover, all patients submitted to secondary cytoreductive surgery were also submitted to hyperthermic intraperitoneal chemotherapy. Although multiple surgical procedures had been performed in order to increase the maximal debulking effort, a single case required reoperation due to the development of an early postoperative hemoperitoneum; as for the diaphragmatic surgery related complications, the most commonly reported ones consisted of the apparition of pleural effusion (reported in $42.5 \%$ of cases). However, only three cases necessitated performing a thoracocentesis and other seven cases necessitated the placement of a chest tube, in all the other cases the pleural effusion being successfully resolved in a conservative management. In univariate analysis, the presence of pleural effusion was significantly correlated with complete liver mobilization and with the presence of large diaphragmatic lesions (lesions larger than $5 \mathrm{~cm}$ ); interestingly, neither the time of debulking (primary, interval debulking or secondary cytoreductive surgery) nor the association of hyperthermic intraperitoneal chemotherapy affected the rate of the apparition of pleural effusion. Moreover, the authors concluded that there was no early postoperative mortality, demonstrating that diaphragmatic surgery can be safely associated with other upper abdominal surgical procedures as well as with intraperitoneal chemotherapy in both primary and relapsed ovarian cancer. When it came to the necessity of routinely chest tube placement, the same study concluded that such drainage procedures should be only performed in cases undergoing complete liver mobilization, extended peritoneal stripping or full thickness diaphragmatic muscle resection. As for the long term outcomes, the overall survival in patients submitted to primary cytoreductive surgery was 30 months while in patients submitted to secondary cytoreductive surgery (from the second surgical procedure) was 24 months (6).

Another frequently reported complication after diaphragmatic resections consists in the apparition of pneumothorax. However, it has been suggested that a meticulous closure of the pleura in association with maximal lung expansion and negative intrapleural pressure represents the best option in order to decrease the rate of postoperative pneumothorax (5).

\section{Conclusion}

Diaphragmatic surgery consisting of peritoneal stripping, electrocoagulation of the peritoneal nodules of carcinomatosis or full-thickness resection can be safely associated as part of debulking surgery in order to increase the debulking effort and to improve survival. Moreover, association of other upper abdominal surgical procedures can be also associated without significant increase of the perioperative outcomes.

\section{References}

1 Eisenhauer EL and Chi DS: Liver mobilization and diaphragm peritonectomy/resection. Gynecol Oncol 104: 25-28, 2007.

2 Einenkel J, Ott R, Handzel R, Braumann UD and Horn LC: Characteristics and management of diaphragm involvement in patients with primary advanced-stage ovarian, fallopian tube, or peritoneal cancer. Int J Gynecol Cancer 19: 1288-1297, 2009.

3 Zapardiel I, Peiretti M, Zanagnolo V, Biffi R, Bocciolone L, Landoni F, Aletti G, Colombo N and Maggioni A: Diaphragmatic surgery during primary cytoreduction for advanced ovarian cancer: peritoneal stripping versus diaphragmatic resection. Int J Gynecol Cancer 21: 1698-1703, 2011.

4 Chereau E, Ballester M, Lesieur B, Selle F, Coutant C, Rouzier $\mathrm{R}$ and Darai E: (Complications of radical surgery for advanced ovarian cancer). Gynecol Obstet Fertil 39: 21-27, 2011.

5 Soleymani MH, Ferrari F, Manek S, Gubbala K, Campanile RG, Hardern K and Tozzi R: Diaphragmatic peritonectomy vs. full thickness resection with pleurectomy during Visceral-Peritoneal Debulking (VPD) in 100 consecutive patients with stage IIICIV ovarian cancer: A surgical-histological analysis. Gynecol Oncol 140: 430-435, 2016.

6 Fanfani F, Fagotti A, Gallotta V, Ercoli A, Pacelli F, Costantini B, Vizzielli G, Margariti PA, Garganese G and Scambia G: Upper abdominal surgery in advanced and recurrent ovarian cancer: role of diaphragmatic surgery. Gynecol Oncol 116: 497$501,2010$.

7 Bristow RE, Tomacruz RS, Armstrong DK, Trimble EL and Montz FJ: Survival effect of maximal cytoreductive surgery for advanced ovarian carcinoma during the platinum era: a metaanalysis. J Clin Oncol 20: 1248-1259, 2002.

8 Bacalbasa N, Dima S, Balescu I, David L, Brasoveanu V and Popescu I: Results of primary cytoreductive surgery in advanced-stage epithelial ovarian cancer: A single-center experience. Anticancer Res 35: 4099-4104, 2015.

9 Bacalbasa N, Balescu I, Dima S, Herlea V, David L, Brasoveanu $\mathrm{V}$ and Popescu I: Initial incomplete surgery modifies prognosis in advanced ovarian cancer regardless of subsequent management. Anticancer Res 35: 2315-2320, 2015.

10 Bacalbasa N, Balescu I, Dima S, Brasoveanu V and Popescu I: Splenectomy as part of cytoreductive surgery in recurrent epithelial ovarian cancer. Anticancer Res 35: 5097-5101, 2015.

11 Bacalbasa N, Balescu I, Dima S, Brasoveanu V and Popescu I: Pancreatic resection as part of cytoreductive surgery in advanced-stage and recurrent epithelial ovarian cancer - A single-center experience. Anticancer Res 35: 4125-4129, 2015.

12 Shih KK and Chi DS: Maximal cytoreductive effort in epithelial ovarian cancer surgery. J Gynecol Oncol 21: 75-80, 2010 
13 Bacalbasa N, Dima S, Brasoveanu V, David L, Balescu I, Purnichescu-Purtan R and Popescu I: Liver resection for ovarian cancer liver metastases as part of cytoreductive surgery is safe and may bring survival benefit. World J Surg Oncol 13: 235, 2015.

14 Eisenkop SM and Spirtos NM: Procedures required to accomplish complete cytoreduction of ovarian cancer: is there a correlation with "biological aggressiveness" and survival? Gynecol Oncol 82: 435-441, 2001.

15 Bacalbasa N, Balescu I, Dima S, Brasoveanu V and Popescu I: Hematogenous splenic metastases as an independent negative prognosis factor at the moment of primary cytoreduction in advanced stage epithelial ovarian cancer - A single center experience. Anticancer Res 35: 5649-5654, 2015.

16 Bacalbasa N, Balescu I, Dima S and Popescu I: Long-term survivors after liver resection for ovarian cancer liver metastases. Anticancer Res 35: 6919-6923, 2015.

17 Muallem MZ, Almuheimid J, Richter R, Braicu EI, Osman S and Sehouli J: Diaphragmatic surgery in advanced ovarian, tubal and peritoneal cancer. A 7-year retrospective analysis of the tumor bank ovarian cancer network. Anticancer Res 36: 4707-4713, 2016.

18 Bacalbasa N, Balescu I, Dima S and Popescu I: Hepatic resection for liver metastases from cervical cancer is safe and may have survival benefit. Anticancer Res 36: 3023-3027, 2016.
19 Gouy S, Chereau E, Custodio AS, Uzan C, Pautier P, HaieMeder C, Duvillard P and Morice P: Surgical procedures and morbidities of diaphragmatic surgery in patients undergoing initial or interval debulking surgery for advanced-stage ovarian cancer. J Am Coll Surg 210: 509-514, 2010.

20 Vergote I, Tropé CG, Amant F, Kristensen GB, Sardi JE, Ehlen T, Johnson N, Verheijen RHM, Van der Brug MEL, Lacave AJ, Benedetti Panici P, Kenter GG, Casado A, Mendiola C, Coens C, Stuart G, Pecorelli S and Reed NS: EORTC-GcG/NCIC-CTG Randomised trial comparing primary debulking surgery with neoadjuvant chemotherapy in stage IIIC-IV ovarian, fallopian tube and peritoneal cancer (OVCA). Presented at the 12th Biennial Meeting International Gynecologic Cancer Society (IGCS) edition:12, Bangkok, Thailand, 25-28 Oct 2008.

Received December 1, 2017

Revised January 25, 2018

Accepted January 29, 2018 